\title{
Probing the effects of hadronic acceleration at the SN 1006 shock front
}

\author{
Marco Miceli $^{1}$, F. Bocchino ${ }^{1}$, A. Decourchelle ${ }^{2}$, G. Maurin ${ }^{3}$, J. Vink $^{4}$, \\ S. Orlando ${ }^{1}$, F. Reale ${ }^{5,1}$ and S. Broersen ${ }^{4}$ \\ ${ }^{1}$ INAF-Osservatorio Astronomico di Palermo, Piazza del Parlamento 1, 90134 Palermo, Italy \\ email: miceli@astropa.inaf.it \\ ${ }^{2}$ Service d'Astrophysique/IRFU/DSM, CEA Saclay, Gif-sur-Yvette, France \\ ${ }^{3}$ Université de Savoie, 27 rue Marcoz, BP 1107 73011-Chambery cedex, France \\ ${ }^{4}$ Astronomical Institute "Anton Pannekoek", University of Amsterdam, P.O. Box 94249, 1090 \\ GE Amsterdam, The Netherlands \\ ${ }^{5}$ Dipartimento di Fisica e Chimica, Università di Palermo, Piazza del Parlamento 1, 90134 \\ Palermo, Italy
}

\begin{abstract}
Supernova remnant shocks are strong candidates for being the source of energetic cosmic rays and hadron acceleration is expected to increase the shock compression ratio, providing higher post-shock densities. We exploited the deep observations of the XMM-Newton Large Program on SN 1006 to verify this prediction. Spatially resolved spectral analysis led us to detect X-ray emission from the shocked ambient medium in SN 1006 and to find that its density significantly increases in regions where particle acceleration is efficient. Our results provide evidence for the effects of acceleration of cosmic ray hadrons on the post-shock plasma in supernova remnants.
\end{abstract}

Keywords. X-rays: ISM - ISM: supernova remnants - ISM: individual object: SN 1006

\section{Introduction}

Supernova remnants (SNRs) shocks are efficient sites of particle acceleration and are candidates for being the main source of the observed spectrum of cosmic rays up to at least $3 \times 10^{15} \mathrm{eV}$ (Berezhko \& Völk 2007). X-ray synchrotron emission from high energy electrons accelerated at the shock front up to $\mathrm{TeV}$ energies has been first observed in SN 1006 (Koyama et al. 1995) and then in other young SNRs as, for example, G1.9+0.3 (Reynolds et al. 2009), Vela Jr. (Slane et al. 2001, Bamba et al. 2005), and G353.6-0.7 (Tian et al. 2010). Recently, TeV emission has been detected in SN 1006 (Acero et al. 2010). The origin of the gamma-ray emission can be leptonic (i.e. inverse Compton from the accelerated electrons) or hadronic (i.e. proton-proton interactions with $\pi^{0}$ production and subsequent decay). The hadronic scenario would directly prove that SNRs can accelerate cosmic-rays up to $\mathrm{PeV}$ energies. Unfortunately, it is not easy to unambiguously ascertain the origin of the $\mathrm{TeV}$ emission. In fact, though a pure leptonic model is consistent with the SN 1006 observations, a mixed scenario that includes leptonic and hadronic components also provides a good fit to the data Acero et al. 2010.

An alternative way to reveal hadron acceleration in SNRs is to probe its effects on the shock dynamics. The loss of energy related to the acceleration of high-energy particles and their non-linear back-reaction on the background plasma are in fact predicted to modify the shock properties, by making the shock compression ratio higher and the post-shock temperature lower than that expected from the Rankine-Hugoniot equations (Berezhko \& Ellison 1999, Decourchelle et al. 2000, Blasi 2002, Vink et al. 2010). This is known 
as "shock modification". The observational confirmation of these predictions requires accurate diagnostics of the thermal X-ray emission from the shocked interstellar medium (ISM). However, the ISM contribution in the X-ray spectra of remnants where particle acceleration is efficient is typically masked-out by the bright synchrotron emission and by the thermal emission from shocked ejecta, as in RXJ1713.7-3946 (Acero et al. 2009), Vela Jr. (Pannuti et al. 2010), G1.9+0.3 (Borkowski et al. 2010), and Tycho (Cassam-Chenaï et al. 2007).

SN 1006 exhibits a morphology characterized by two opposed radio, X-ray, and $\gamma$-ray bright limbs dominated by non-thermal emission, separated by a region at low surface brightness with soft, thermal X-ray emission. Thermal X-ray emission has been associated with shocked ejecta (Acero et al. 2007, Miceli et al. 2009). Indirect evidence for shock modification in SN 1006 has been obtained by measuring the distance, $D_{B W C D}$, between the shock front and the contact discontinuity that is expected to be smaller in nonthermal limbs, where particle acceleration is more efficient. $D_{B W C D}$ is instead almost the same all over the shell (even in regions dominated by thermal emission), though it is much smaller than that expected from unmodified shocks (Cassam-Chenaï et al. 2008, Miceli et al. 2009). 3-D magneto-hydrodynamic simulations have recently shown that this small distance can be naturally explained by ejecta clumping, without invoking shock modification (Orlando et al. 2012). Therefore, the small value of $D_{B W C D}$ is not a reliable indicator of hadronic acceleration.

We took advantage of new observations within the XMM-Newton SN 1006 Large Program (PI A. Decourchelle, $700 \mathrm{ks}$ of total exposure time) to present the first robust detection of X-ray emission from shocked ISM in SN 1006 and show evidence for the effects of hadron acceleration on the post-shock plasma (see Miceli et al. 2012 for further details). The bilateral morphology of SN 1006 clearly reveals regions with high injection efficiency, $\eta \dagger$, (i.e. the limbs), separated by regions with low $\eta$. We therefore expect to observe stronger shock modification near the non-thermal limbs than in the thermal regions.

\section{Results}

We first focused on the center of a thermal rim (region $e$ in Fig. 1) where the contribution of the synchrotron emission is the smallest and we expected minimum shock modification. We have found that with the new data, a model with three components (ejecta, ISM, and synchrotron emission) fits the spectrum of region $e$ significantly better than a model with only two components. For example, a thermal component for the ejecta (optically thin plasma in non-equilibrium of ionization with free $\mathrm{O}, \mathrm{Ne}, \mathrm{Mg}$, and $\mathrm{Si}$ abundances, VPSHOCK model in XSPEC, Borkowski et al. 2001), plus the non-thermal SRCUT component (Reynolds \& Keohane 1999), yields a $\chi^{2}=1199.8$ (with 658 d. o.f.), while by adding another VPSHOCK component with solar abundances, we get a much lower value $\left(\chi^{2}=1112.0\right.$ with 655 d. o .f.). The normalization of the additional component is larger than zero at $>11$ sigmas (more details of spectral analysis are provided in Miceli et al. 2012). The association of the additional thermal component with shocked ISM is supported by its parameters, derived from spectral analysis: i) the post-shock density $n_{I S M}=0.14 \pm 0.01 \mathrm{~cm}^{-3}$ indicates a pre-shock density $<0.05 \mathrm{~cm}^{-3}$, consistent with the high galactic latitude of the remnant and in agreement with the upper limit present in the literature (Acero et al. 2007); ii) the ionization parameter (i.e. the integral of density over time calculated from the impact with the shock front) $\tau_{I S M} \sim 7 \times 10^{8}$

$\dagger \eta$ is the fraction of particles injected in the acceleration process. 


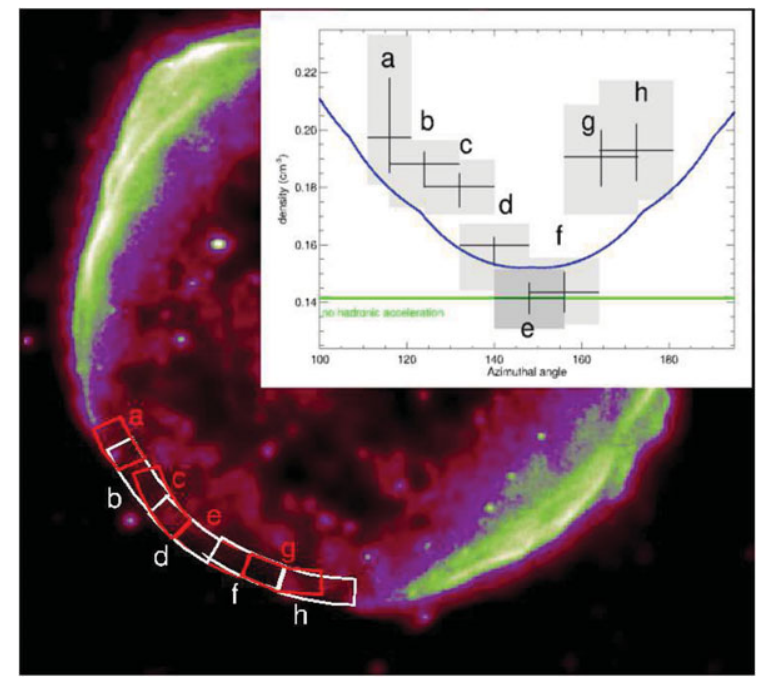

Figure 1. XMM-Newton image of SN 1006 in the $2-4.5 \mathrm{keV}$ band (the bin size is $4^{\prime \prime}$ ). The inset shows the azimuthal profile of the ISM density in the spectral regions. Error bars are at the $68 \%$ (crosses) and $90 \%$ (shaded areas) confidence levels. The blue curve shows the profile derived by assuming that $\eta$ is proportional to the radio flux divided by $B^{3 / 2}$, and adopting the magnetic field model MF2 of Petruk et al. 2009 and by assuming the relationship between the injection efficiency and shock compression ratio adopted by Ferrand et al. 2010.

$\mathrm{s} \mathrm{cm}^{-3}$ provides a very reasonable estimate for the time elapsed after the shock impact $(\sim 200 \mathrm{yr})$, considering the $1 \mathrm{kyr}$ age of the remnant; and iii) the temperature $\left(k T_{I S M} \sim\right.$ $1.4 \mathrm{keV}$ ) suits the expectations for the shocked ISM, as shown below. In collisionless shocks at high Mach number (shock velocity of the order of $10^{3} \mathrm{~km} / \mathrm{s}$ ) the electron to proton temperature ratio is expected to be $T_{e} / T_{p} \ll 1$ (Ghavamian et al. 2007, Vink et al. 2003). In the north-western limb of SN 1006 the shock velocity is $v_{s h} \sim 3000 \mathrm{~km} / \mathrm{s}$ and it has been calculated that $T_{e} / T_{p}<0.07$ (Ghavamian et al. 2002). Considering that in our south-eastern region the shock speed is $\sim 5000 \mathrm{~km} / \mathrm{s}$ (Katsuda et al. 2009) and assuming the same ratio (and no or little shock modification) we get $k T_{e}<3.5 \mathrm{keV}$, in agreement with our findings.

If efficient hadron acceleration is at work, we expect to observe a higher shock compression ratio and a lower shock temperature in regions closer to the non-thermal limbs. The modeling of our spectra allows us to measure the electron temperature only and it is still unclear how to couple $T_{e}$ with the shock temperature (Vink 2012). We therefore consider as the most reliable indicators of hadron acceleration the variations in the ISM post-shock density that can be directly derived through our spectral analysis. To verify the presence of shock modification, we then analyzed the spectra extracted from regions $[a, b, c, d]$, and $[f, g, h]$ (shown in Fig. 1) that are closer than $e$ to the non-thermal limbs (more details in Miceli et al. 2012). In all these regions we confirmed the detection of the ISM component. We found that the post-shock density significantly increases in regions where particle acceleration is more efficient (see inset in Fig. 1). Considering that the pre-shock density is expected to be fairly uniform (Dubner et al. 2002), the density profile in Fig. 1 shows that the shock compression ratio is higher near the non-thermal limbs, thus providing a direct proof for the shock modification theory.

We also compared the observed ISM density profile with that obtained by assuming that $\eta$ is proportional to the radio flux divided by $B^{3 / 2}$, and adopting the magnetic field model MF2 of Petruk et al. 2009 (the relationship between the injection efficiency and 
the shock compression factor has been obtained by following Ferrand et al. 2010). The green line in Fig. 1 shows the constant compression ratio scenario that would result for no shock modification.

It was not possible to extend our analysis by looking at regions closer to (or inside) the non-thermal limbs. In fact, the significance of the detection of the ISM component drops down as the synchrotron contribution increases. This result confirms that the additional ISM component has indeed a thermal origin and is not an artifact due to a misdescription of the synchrotron emission (in this case we should have detected it with higher statistical significance in synchrotron-dominated regions).

In conclusion, we have obtained a firm detection of the X-ray emission from the shocked ISM in SN 1006 and found evidence for the presence of shock modification induced by hadron acceleration at the shock front. Our results provide new constraints for future theories and prove that cosmic ray acceleration is present at the shock front and modifies the shock properties.

\section{References}

Acero, F., Aharonian, F., Akhperjanian, A. G., et al. 2010, A\&A, 516, A62

Acero, F., Ballet, J., \& Decourchelle, A. 2007, A\&A, 475, 883

Acero, F., Ballet, J., Decourchelle, A., et al. 2009, A\&A, 505, 157

Bamba, A., Yamazaki, R., \& Hiraga, J. S. 2005, ApJ, 632, 294

Berezhko, E. G. \& Ellison, D. C. 1999, ApJ, 526, 385

Berezhko, E. G. \& Völk, H. J. 2007, АpЛ, 661, L175

Blasi, P. 2002, Astroparticle Physics, 16, 429

Borkowski, K. J., Lyerly, W. J., \& Reynolds, S. P. 2001, ApJ, 548, 820

Borkowski, K. J., Reynolds, S. P., Green, D. A., et al. 2010, ApJ, 724, L161

Cassam-Chenaï, G., Hughes, J. P., Ballet, J., \& Decourchelle, A. 2007, ApJ, 665, 315

Cassam-Chenaï, G., Hughes, J. P., Reynoso, E. M., Badenes, C., \& Moffett, D. 2008, ApJ, 680, 1180

Decourchelle, A., Ellison, D. C., \& Ballet, J. 2000, ApЛ, 543, L57

Dubner, G. M., Giacani, E. B., Goss, W. M., Green, A. J., \& Nyman, L.-Å. 2002, A $₫ A, 387$, 1047

Ferrand, G., Decourchelle, A., Ballet, J., Teyssier, R., \& Fraschetti, F. 2010, A\& A, 509, L10+

Ghavamian, P., Laming, J. M., \& Rakowski, C. E. 2007, ApJ, 654, L69

Ghavamian, P., Winkler, P. F., Raymond, J. C., \& Long, K. S. 2002, ApJ, 572, 888

Katsuda, S., Petre, R., Long, K. S., et al. 2009, ApJ, 692, L105

Koyama, K., Petre, R., Gotthelf, E. V., et al. 1995, Nature, 378, 255

Miceli, M., Bocchino, F., Decourchelle, A., et al. 2012, A\& A, 546, A66

Miceli, M., Bocchino, F., Iakubovskyi, D., et al. 2009, A\&A, 501, 239

Orlando, S., Bocchino, F., Miceli, M., Petruk, O., \& Pumo, M. L. 2012, ApJ, 749, 156

Pannuti, T. G., Allen, G. E., Filipović, M. D., et al. 2010, ApJ, 721, 1492

Petruk, O., Bocchino, F., Miceli, M., et al. 2009, extitMNRAS, 399, 157

Reynolds, S. P., Borkowski, K. J., Green, D. A., et al. 2009, ApЛ, 695, L149

Reynolds, S. P. \& Keohane, J. W. 1999, ApJ, 525, 368

Rothenflug, R., Ballet, J., Dubner, G., et al. 2004, A\&A, 425, 121

Slane, P., Hughes, J. P., Edgar, R. J., et al. 2001, ApJ, 548, 814

Tian, W. W., Li, Z., Leahy, D. A., et al. 2010, ApJ, 712, 790

Vink, J. 2012, A\&Ar, 20, 49

Vink, J., Laming, J. M., Gu, M. F., Rasmussen, A., \& Kaastra, J. S. 2003, ApЛ, 587, L31

Vink, J., Yamazaki, R., Helder, E. A., \& Schure, K. M. 2010, ApJ, 722, 1727

Yamaguchi, H., Koyama, K., Katsuda, S., et al. 2008, PASJ, 60, 141 


\section{Discussion}

Editor's Note: The paper was presented by Fabrizio Bocchino. The discussion reproduced here took place after Bocchino's (separate) talk on "X-ray emission from ejecta fragments and protrusions in and around the SN1006 shell" which was on a related topic on the same SNR. No manuscript was received from Dr. Bocchino on his paper.

Folatelli: Do you have an explanation for the protrusions that are 'outliers' in terms of size as compared with your models?

BocCHINO: We are looking into those. An expansion inside a more rarefied medium could be a possibility.

LU: Is there any difference along the lines from the center to the protrusions from the other regions of the remnant just inside the shock? (any sign of the fast moving fragments?)

Bocchino: We haven't investigated this issue yet. The limited-time span between XMMNewton observations and the moderate spatial resolution make proper motion studies difficult.

BRANDT: What might we learn from regions of negative protrusions ?

Bocchino: "Negative Protrusions" are regions where the ejecta have not reached the main shock. They are useful in the sense that both "negative" and "regular" protrusion concur to establish the azimuthal periodicity seen in the data. They also concur to establish a mean $R_{C D} / R_{B W}$ ratio which may be compared to models.

Chiotellis: Do the ejecta protrusion affect the overall kinematics of the forward shock ? Do they also affect the shocked ejecta shell properties?

Bocchino: No, they don't, because perturbations of ejecta profiles are done maintaining the total kinetic energy constant, and moreover the density clumps are very few. So the overall dynamic is not significantly affected.

SLANE: The modeling here makes it seem clear that clumpy ejecta are required to get protrusions beyond the FS. However, we know there is CR acceleration too, so it isn't clear why this has been eliminated in the Final Modeling. Both effects must be playing a role in compressing the $\mathrm{FS} / \mathrm{CD}$ ratio, right?

Bocchino: The poster of Orlando (already published on astro-ph) shows CR acceleration has only a second order effects on protrusions and $R_{B W} / R_{C D}$ ratio. So it has been eliminated for simplicity in this work. Yes, CRs indeed do play a major role in compressibility and we show in the Miceli talk that this effect is measurable in SN1006.

SCHURE: On what is your initial clumpiness based and is there any information from explosion models that can shed light on this? It could well be that they are not homogeneously distributed?

Bocchino: The initial clumpiness is arbitrary, but realistic. We plan to improve it by coupling our simulation with explosion models having clumpiness. Yes, it could well be these are not homogeneously distributed. 